\title{
Morphogenèse, acide rétinoïque... et Sonic Hedgehog
}

Les interactions entre tissus par le biais desquelles un tissu agit sur le développement d'un tissu voisin, ou inductions, jouent un rôle crucial au cours de l'ontogenèse. C'est l'embryologie expérimentale, greffant ou extirpant tel ou tel tissu, qui l'a établi de manière classique [1] ; mais, à l'exception peut-être du cas de l'induction mésodermique [2], les mécanismes et molécules impliqués dans ces processus inductifs restent énigmatiques chez les vertébrés [3]. Chez la drosophile, la recherche systématique de mutants affectant le diagramme de segmentation a permis d'identifier un grand nombre de gènes du développement [4]. En particulier, sont connus des gènes qui contrôlent les premières interactions cellulaires' régissant l'organisation antéro-postérieure des segments de l'embryon, dits gènes de polarité segmentaire. Le plus célèbre à ce jour est le gène wingless, car la découverte de son homologie avec le protooncogène murin Wnt-1 avait révélé pour la première fois les liens entre gènes du cancer et gènes du développement $[5,6]$. Par la suite, il a été montré que le gène Wnt-1, à l'instar de son homologue wingless, est essentiel au développement [5]. Mais un gène de polarité segmentaire peut en cacher un autre...

Pour identifier les gènes de messagers des inductions ayant lieu au cours de l'ontogenèse, il est donc possible de rechercher des homologues de gènes contrôlant les interactions cellulaires chez la drosophile. Suivant cette démarche, trois équipes viennent de montrer que le gène hedgehog, cloné fin 1992 chez

homologues chez les vertébrés et que l'un d'eux, Sonic hedgehog, joue un rôle clé dans les inductions responsables de l'organisation spatiale du système nerveux central (SNC) et des membres [9-11].

Le gène hedgehog : un signal intercellulaire essentiel au développement de la drosophile

Au cours de la croissance de la larve de drosophile, le maintien des frontières parasegmentaires et, par ce biais, celui de l'organisation spatiale de l'embryon, reposent sur les interactions entre les cellules de part et d'autre de la frontière entre parasegments voisins. D'un côté, les cellules agissent sur leur voisines par l'intermédiaire de wingless, et de l'autre, ces dernières agissent en retour sur les premières par l'intermédiaire de... hedgeghog [12]. Les mutations de hedgehog initialement caractérisées désorganisent totalement l'arrangement des denticules de l'épiderme larvaire, lui donnant une allure de hérisson (hedgehog en anglais) .

Hedgehog participe en fait à de nombreux événements morphogénétiques du développement de la drosophile : le déplacement du sillon à travers le disque oculaire, dont le passage déclenche la différenciation de cet épithélium et constitue une étape clé de la formation de l'œil [13, 14] ; l'organisation suivant l'axe proximo-distal des cellules des disques imaginaux [15] ; la différenciation de l'épiderme larvaire [16]. Dans tous les cas où elle a été réalisée, l'analyse de mosaïques génétiques montre que les cellules non mutantes voisines des clones cellulaires hedgehog -/- sont affectées, et par conséquent que hedgehog code pour un signal intercellulaire [13, 17]. La protéine est effectivement localisée à l'extérieur des cellules qui la synthétisent, mais elle semble toutefois rester principalement dans leur proximité immédiate [15, 18].

Sonic hedgehog est exprimé dans plusieurs tissus inducteurs au cours du développement des vertébrés

Trois équipes ont décrit l'existence d'homologues du gène hedgehog chez la souris, le poisson-zèbre, le poulet et le xénope ([9-11] et J-P. C., résultats non publiés). Dans toutes ces espèces, un même homologue, appelé Sonic hedgehog d'après le jeu électronique fabriqué par Sega (sic), a été identifié et étudié en détail, mais d'autres homologues ont été découverts et la famille pourrait comporter de nombreux autres membres. Dans tous les cas, l'extrémité amino-terminale des protéines codées par ces gènes présente un domaine hydrophobe d'une vingtaine d'acides aminés, suivi d'un site de clivage potentiel, structure caractéristique des protéines secrétées. La signification de la conservation de séquence observée a été renforcée par la démonstration d'une conservation fonctionnelle: le gène Sonic hedgehog de poisson-zèbre possède les mêmes propriétés que hedgehog dans des expériences d'expression ubiquiste chez la drosophile [10]. Pour identifier les fonctions possibles de Sonic hedgehog, les sites d'expression de ce gène ont été examinés au cours du développement. 
Peu après le début de la gastrulation, la transcription de Sonic hedgehog débute dans le centre organisateur [2] ; au cours de la somitogenèse et de la neurulation, elle se poursuit dans ses dérivés, le mésoderme axial puis la notochorde, et est alors activée dans le SNC ventral de manière rostro-caudale (territoire qui correspond au plancher du tube nerveux à partir du mésencéphale), cette activation étant suivie de l'extinction dans la notochorde sousjacente. Enfin, lors de l'apparition des bourgeons de membres (de nageoires chez le poisson!), Sonic hedgehog est transcrit dans une région postérieure de leur mésenchyme.

Notochorde, plancher du tube nerveux, mésenchyme postérieur des bourgeons de membres: tous ces tissus possèdent d'importantes propriétés inductrices. Et de manière tout à fait remarquable, ces trois tissus peuvent être utilisés indifféremment dans les expériences démontrant leurs propriétés respectives; la notochorde et le plancher du tube nerveux provoquent des duplications en miroir des doigts de poulet s'ils sont greffés dans la partie antérieure des bourgeons de membres [19] tandis que le mésenchyme postérieur des bourgeons de membres provoque l'induction d'un plancher ectopique dans des explants du tube nerveux latéral cultivés à son contact $[20] \ldots$ Sonic hedgehog, qui est très probablement une protéine secrétée, est exprimée dans ces trois tissus, devenant ainsi un candidat de choix pour le rôle de messager de leur pouvoir inducteur.

\section{Polarisation dorso-ventrale du SNC et Sonic hedgehog}

L'apparition et l'organisation spatiale du SNC procèdent par induc-

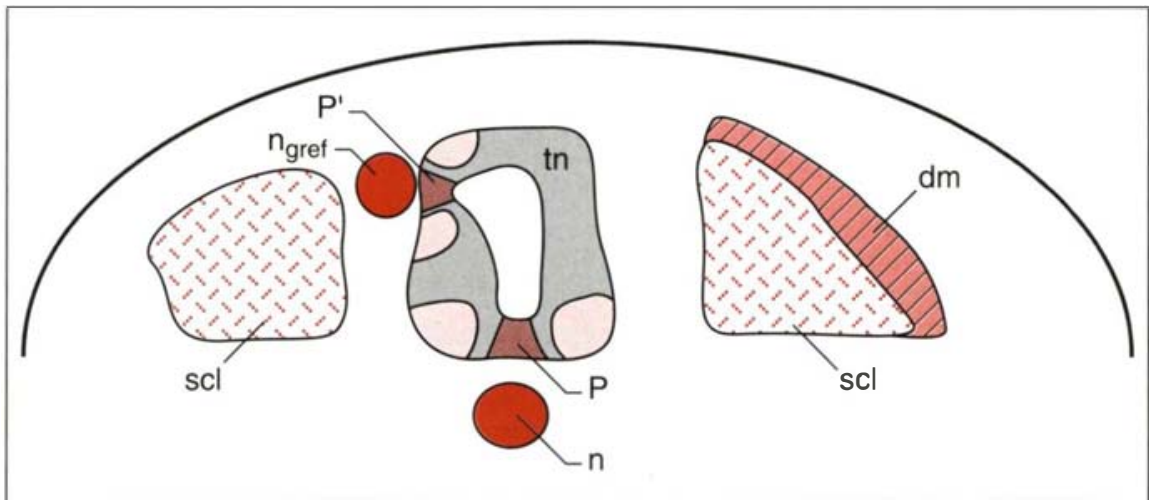

Figure 1. Polarisation dorso-ventrale du tube nerveux et des somites par la notochorde et le plancher du tube nerveux. Le rôle de la notochorde et du plancher du tube nerveux dans la polarisation dorso-ventrale du tube nerveux et des somites a été déduit de l'analyse d'expériences de greffe ou d'ablation de ces tissus [21-23]. Le schéma d'une section transversale d'embryon de poulet obtenu après la greffe d'une notochorde supplémentaire est représenté. La greffe d'une notochorde supplémentaire (ngref) le long du tube nerveux d'un embryon-hôte provoque une "ventralisation" des tissus adjacents: dans le tube nerveux, un plancher ectopique $\left(P^{\prime}\right)$ apparaît dans le tube nerveux latéral en contact avec la notochorde greffée et des motoneurones ectopiques apparaissent de part et d'autre de ce plancher ectopique [21, 22]; dans le somite adjacent, une différenciation sclérotomale prédomine [23]. Le côté droit de l'embryon possède une organisation dorso-ventrale normale. Des résultats similaires sont obtenus après la greffe d'un plancher du tube nerveux supplémentaire [21-23]. (D'après Pourquié et al. [23]) $n$ : notochorde; $p$ : plancher du tube nerveux; tn: tube nerveux; dm: dermamyotome; scl: sclérotome; les zones hachurées contiennent des motoneurones.

$\mathrm{m} / \mathrm{s} n^{\circ} 5$ vol. 10, mai 94 tions successives. Ainsi, l'ectoderme indifférencié, produit durant la gastrulation, donne naissance au cerveau et au tube nerveux sous l'effet d'inductions par le centre organisateur et le mésoderme axial [1].

Dans le tube nerveux, l'apparition de l'organisation dorso-ventrale est bien décrite. La notochorde, dérivée du mésoderme axial, et le plancher du tube nerveux y jouent un rôle prédominant (figure 1). La notochorde entraîne la différenciation du plancher du tube nerveux; puis, à partir de signaux émis par la notochorde et ce même plancher, le tube nerveux s'organise et se différencie: les motoneurones apparaissent en position ventro-latérale de part et d'autre du plancher, des axones commissuraux se dirigent vers le plancher, ... [21, 22]. Pour examiner le rôle de Sonic hedgehog au cours du développement du tube nerveux, des expériences d'expression ectopique ont été réalisées. En forçant l'expression de Sonic hedgehog à des positions ectopiques dans l'embryon de souris (au moyen d'un transgène exprimant Sonic hedgehog sous le contrôle des séquences régulatrices du gène $W n t-1)$ ou dans l'embryon de poisson-zèbre (par microinjection d'ARNm transcrits in vitro dans l'œuf fécondé), une expression ectopique de marqueurs du plancher a été provoquée, suggérant que les cellules ayant reçu un signal Sonic hedgehog ectopique ont été conduites à adopter des caractéristiques de cellules du plancher du tube nerveux $[9,10]$. Sonic hedgehog serait donc le médiateur de l'induction du plancher du tube nerveux. Toutefois, l'expression ectopique de marqueurs du plancher qui a été décrite est restreinte à certaines régions du domaine d'expression ectopique de Sonic hedgehog. En particulier, elle n'est pas observée dans le tube nerveux qui, d'après les expériences de greffe de notochorde ou de plancher, sont tout à fait aptes à répondre à ces inducteurs du plancher. Il est donc possible que Sonic hedgehog ne soit pas à lui seul le médiateur des activités de la notochorde et du plancher du tube nerveux; un autre fac- 
teur (ou plusieurs) pourrait être nécessaire. On peut, par ailleurs, noter que la notochorde et le plancher du tube nerveux partagent également la propriété d'agir sur la polarisation dorso-ventrale des somites (figure 1) [23] et la participation de Sonic hedgehog à ce processus est donc envisageable.

Polarisation dorso-ventrale du cerveau et Sonic hedgehog

Dans le cerveau, contrairement à ce qui se passe dans le tube nerveux, l'apparition de l'organisation dorsoventrale est mal comprise. Cependant, et c'est tout à fait remarquable, l'expression de Sonic hedgehog dans le SNC ventral s'étend en fait antérieurement au-delà du plancher. L'organisation dorso-ventrale du cerveau pourrait donc en partie reposer sur des molécules (Sonic hedgehog) et des mécanismes analogues à ceux employés dans le tube nerveux. En particulier, le territoire ventral du cerveau pourrait donc avoir des propriétés communes avec le plancher. L'importance de ce territoire dans l'organisation du cerveau est soulignée par le phénotype du mutant de poisson-zèbre cyclops [24]. Chez ce mutant, l'expression de Sonic hedgehog dans le SNC ventral n'apparaît jamais, ni dans le cerveau, ni dans le tube nerveux: c'est la première explication moléculaire de l'absence d'apparition, chez ce mutant, du territoire ventral du SNC [10]. Cette absence du SNC ventral bouleverse l'organisation du cerveau: elle conduit à la délétion d'une grande partie du diencéphale et, par suite, à la cyclopie qui donne son nom à ce mutant. En effet, l'introduction de cellules sauvages cyclops $+/+$ dans un embryon mutant cyclops -/- peut supprimer la cyclopie et, de manière générale, restaurer une organisation normale du cerveau antérieur si des cellules cyclops $+/+$, même en très petit nombre, viennent à se localiser dans le SNC ven-

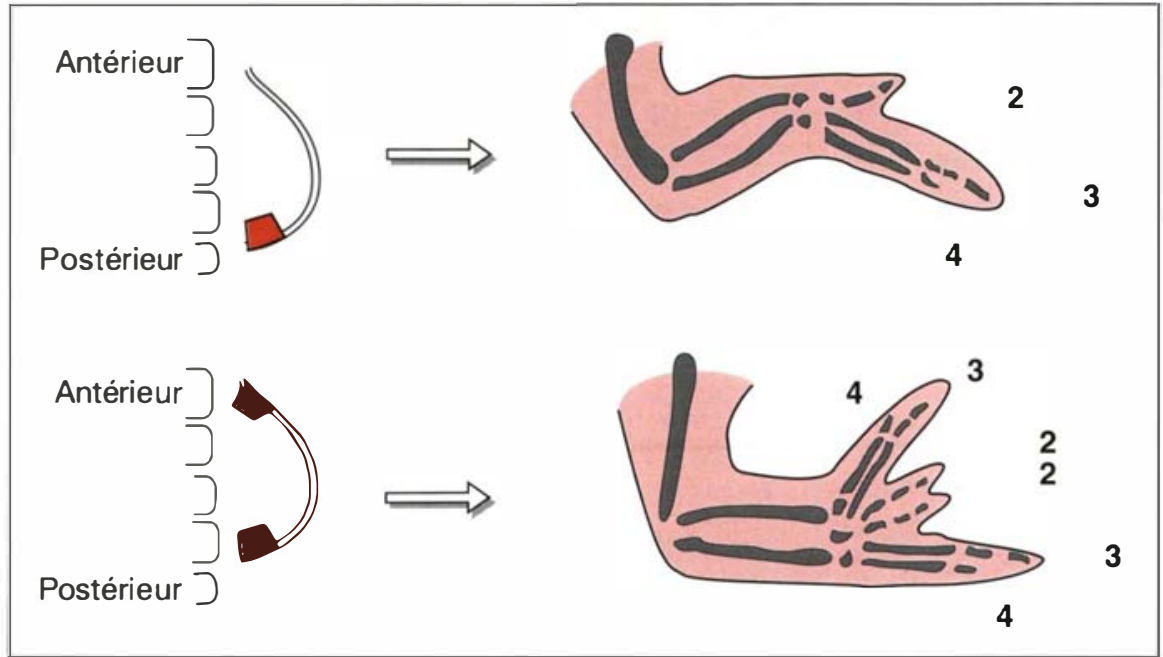

Figure 2. Polarisation antéro-postérieure des membres par le mésenchyme postérieur des bourgeons de membres. Le mésenchyme postérieur des bourgeons de membres correspondant à la zone à activité polarisatrice est représenté en rouge. Partie supérieure de la figure: dans le bourgeon de membre, une polarisation antéro-postérieure est mise en place, ce qui conduit à l'émergence de doigts distincts numérotés de 2 à 4 suivant leur position le long de l'axe antéro-postérieur. Partie inférieure de la figure: la greffe du mésenchyme postérieur d'un bourgeon de membre dans la partie antérieure d'un bourgeon de membre d'un embryon-hôte tral [24]! Peut-être Sonic hedgehog est-il le signal impliqué?

\section{L'acide rétinoïque, morphogène} déchu

L'organisation antéro-postérieure des membres, en particulier l'apparition ordonnée des différents doigts, semble dépendre de l'activité d'une région du mésenchyme postérieur des bourgeons de membres appelée zone à activité polarisatrice. En effet, la greffe de cellules du mésenchyme postérieur des bourgeons de membres dans la partie antérieure d'un bourgeon hôte provoque une spectaculaire duplication en miroir de l'arrangement des doigts (figure 2) [1].

La greffe de deux autres tissus est capable de provoquer cette duplication... : la notochorde et le plancher du tube nerveux [19] ! Cette observation a donné son poids à l'hypothèse d'un rôle de Sonic hedgehog dans ce processus.

Pour tester son rôle dans la polarisation antéro-postérieure des membres, des cellules exprimant Sonic hedgehog après infection rétrovirale ont été implantées dans la partie antérieure de bourgeons de membres: elles provoquent des duplications [11] ! Ces duplications ressemblent remarquablement à celles produites par greffe de la zone à activité polarisatrice. Des duplications de structures osseuses proximales, l'humérus et le radius, ont aussi été observées, suggérant que la polarisation antéro-postérieure des structures proximales des membres est également affectée par l'expression de Sonic hedgehog.

Le modèle le plus populaire pour rendre compte de l'organisation antéro-postérieure des membres fait intervenir un morphogène dont le gradient de concentration serait traduit en information de position par les cellules (modèle dû à Wolpert, [1]). L'implantation d'une bille d'acide rétinoïque est capable de reproduire l'action de la zone à activité polarisatrice et l'acide rétinoïque était donc LE candidatmorphogène. Cependant, d'élégantes manipulations embryologiques 
semblaient aller à l'encontre de cette hypothèse [25] et elle semble désormais définitivement exclue. Riddle $e t$ al. ont en effet montré que l'acide rétinoïque provoque l'activation de Sonic hedgehog. La duplication en miroir des doigts par l'acide rétinoïque est donc certainement une conséquence de cette activation de Sonic hedgehog et non pas de la création d'un nouveau gradient morphogénétique d'acide rétinoïque. Il reste possible que, in vivo, ce soit bien l'acide rétinoïque qui conduise à l'activation de Sonic hedgehog. Cela pourrait intervenir de manière directe, si la transcription du gène est activée par des récepteurs de l'acide rétinoïque, ou, plus probablement, de manière indirecte, compte tenu de la latence observée dans l'apparition de transcrits de Sonic hedgehog après l'implantation de billes d'acide rétinoïque. Mais Sonic. hedgehog est-il alors le morphogène spécifiant une information de position par le biais d'un gradient de concentration? Une description systématique des modifications de la polarité des doigts en fonction de la dose de Sonic hedgehog implantée sera cruciale pour une nouvelle évaluation du modèle de Wolpert. D'autres mécanismes d'action de Sonic hedgehog semblent toutefois envisageables ; par exemple, il pourrait agir au départ d'une cascade d'interactions cellulaires [11].

\section{Quel est le mécanisme de l'action morphogénétique?}

Pour comprendre le mécanisme d'action de Sonic hedgehog, il sera indispensable de déterminer son "rayon d'action" (à quelle distance des cellules sécrétrices la protéine est-elle capable de diffuser? serat-elle toujours active si elle est ancrée à la membrane sous forme d'une chimère avec une protéine transmembranaire [26] ?) et de découvrir les caractéristiques de son récepteur (comment le signal est-il interprété par les cellules réceptrices? comment la compétence à répondre au signal apparaît-elle et comment évolue-t-elle?). Enfin, les travaux chez la drosophile montrent $\mathrm{m} / \mathrm{s} n^{\circ} 5$ vol. 10, mai 94 clairement l'importance de l'association de Sonic hedgehog avec d'autres signaux moléculaires pour la réalisation de ses effets morphogénétiques, partenaires qu'il faudra également identifier chez les vertébrés avant qu'un scénario ne s'impose pour décrire l'action de Sonic hedgehog. Autant de questions qui pourraient permettre une analyse moléculaire de l'apparition des formes et de l'organisation spatiale au cours du développement de l'embryon de vertébré

\section{RÉFÉRENCES}

1. Gilbert SF. Developmental biology. 3rd ed Sinauer associates, Inc, Publishers, 1992. 2. Slack I Inducing factors in Xenopus early embryos. Cur Biol 1994; 4 : 116-26.

3. Gurdon J The generation of diversity and pattern in animal development. Cell 1992: $68: 180-99$.

4. Lepesant JA. Le paradigme de la drosophile dans l'étude du développement. médecine/sciences 1992; 8: 65()-2

5. Mc Mahon AP. The Wnt family of developmental regulators. Trends (ipmet $1992 ; 8$ : 236-42.

6. Nusse R, Varmus H. Wht genes. Cell $1992 ; 69: 1073-97$.

7. Lee II, von Kessler IDP, Parks S, Beachy $P$. Secretion and localized transcription suggest a role in positional information for products of the segmentation gene hedgehog. Cell 1992; 71 : 33-50.

8. Tabata T, Eaton SE, Kornberg TB. The I)rosophila hedgehog gene is expressed specifically in posterior compartment cells and is a target of engrailed regulation. Cones l)ev 1992 ; 6 : 2636-45.

9. Echelard Y, Epstein I), St-Jacques B, Shen 1., Mohler J, Mc Mahon JA, Mc Mahon AP. Sonic hedgehog, a member of a family of putative signaling molecules, is implicated in regulation of (.NS polarity. Cell 1993; 75 : 1417-30

10. Krauss S, Concordet JP, Ingham PW. A functionnaly conserved homolog of the Drosophila segment polarity gene hedgehog is expressed in tissues with polarising activity in zebrafish embryos. Cell 1993; 75 : 1431-45.

1 1. Riddle B, Johnson R, Laufer E, Tabin C. Sonic hedgehog mediates the polarising activity of the ZPA. Cell 1993; 75: 1401-16. 12. Ingham PW, Martinez-Arias A. Boundaries and fields in early embryos. Cell 1992 ; 68 : 221-35

13. Ma C, Zhou Y, Beachy P, Moses K. The segment polarity gene hedgehog is required for progression of the morphogenetic furrow in the developing Drosophila eye. (ell 1993; 75 : 927-38.
14. Heberlein U, Wolff $T$, Rubin (;M. The $T$ (il $\beta$ homolog $d p p$ and the segment polarity gene hedgehog are required for propagation of a morphogenetic wave in the $1 \mathrm{mo}$ sophila retina. Cell 1993; 75 : 913-26.

15. Tabata T, Kornberg, TB. Hedgehog is a signaling protein with a key role in patterning drosophila imaginal discs. Cell $1994: 76$ : 89-102.

16. Heermsek J, diNardo S. Drosophila hedgehog acts as a morphogen in cellular patterning. Cell 1994; $76: 449-60$.

17. Mohler J. Requirements for hedgehog, a segment polarity gene, in patterning larval and adult cuticle of I)rosophila. (ienetics $1988 ; 120$ : 1061-72.

18. Taylor AM, Nakano Y, Mohler J, Ingham P. Contrasting distributions of patched and hedgehog proteins in the I)roso phila embryo. Mech Dev 1993; 42 : 89-96. 19. Wagner M, Thaller (', Jessel T, Eichele (i. Polarizing activity and retinoid synthesis in the floor plate of the neural tube. Nalure 1990 ; 345 : 819-21.

20. Roelink H, Augsburger A, Heemskerk I, Korzh V, Norlin S, Ruiz i Altaba A Tanabe Y, Placzek M, Edlund T, Jessel TM, Dodd J. Floor plate and motor neuron induction by whh-1, a vertebrate homolog of hedgehog expressed by the notochord. Cell 1994; 76: 761-75.

21. Placzek M, Jessel T, Dodd J. Induction of floor plate differentiation by contact dependent, homeogenetic signals. Development 1993; 117: 205-18.

22. Yamada T, Pfaff SI, Edlund T, Jesse $\mathrm{T}$. Control of cell pattern in the neural tube: motor neuron induction by diffusible factors from notochord and floor plate. Cell 1993; 73: 673-86.

23. Pourquié O, Coltey M, Teillet MA, ()rdahl C: I e Douarin NM. Control of dorsoventral patterning of somitic derivatives by notochord and floor plate. Proc Natl Acad Si USA 1993; 90: 5242-6.

24. Hatta K, Puschel AW, Kimmel (BB. Midline signaling in the primordium of the zebrafish anterior central nervous system. Proc Nall Acad Sci USA 1994; 91: 2061-5. 25. Wanek N, Gardiner IDM, Muneoka K, Bryant SV. Conversion by retinoic acid of anterior cells into ZPA cells in the chick wing bud. Nalure 1991 ; 350 : 81-3.

26. Parkin NT, Kitajewski J, Varmus HE. Activity of Wnt-l as a transmembrane protein. Genes Dev 1993; 7 : 2181-93.

Jean-Paul Concordet
Developmental Biology Unit, ICRF,
Department of Zoology, University of
Oxford, Oxford.

TIRÉS A PART

J-P. Concordet. 